Supplement of The Cryosphere, 14, 2313-2330, 2020

https://doi.org/10.5194/tc-14-2313-2020-supplement

(C) Author(s) 2020. This work is distributed under

the Creative Commons Attribution 4.0 License.

(c) (1)

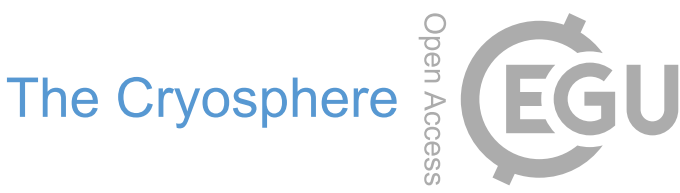

Supplement of

\title{
Lateral meltwater transfer across an Antarctic ice shelf
}

Rebecca Dell et al.

Correspondence to: Rebecca Dell (rld46@cam.ac.uk)

The copyright of individual parts of the supplement might differ from the CC BY 4.0 License. 


\section{Supplements}

\begin{tabular}{|c|c|c|c|}
\hline Image ID & Satellite & $\begin{array}{l}\text { Image } \\
\text { Acquisition } \\
\text { Date }\end{array}$ & Image Group \\
\hline LC08_L1GT_165109_20161102_20170318_01_T2 & Landsat 8 & $02 / 11 / 2016$ & 1 \\
\hline LC08_L1GT_165110_20161102_20170318_01_T2 & Landsat 8 & $02 / 11 / 2016$ & 1 \\
\hline S2A_MSIL1C_20161111T074922_N0204_R106_T32DNG_20161111T074922 & Sentinel-2 & $11 / 11 / 2016$ & 2 \\
\hline S2A_MSIL1C_20161111T074922_N0204_R106_T32DNH_20161111T074922 & Sentinel-2 & $11 / 11 / 2016$ & 2 \\
\hline S2A_MSIL1C_20161111T074922_N0204_R106_T32DPG_20161111T074922 & Sentinel-2 & $11 / 11 / 2016$ & 2 \\
\hline S2A_MSIL1C_20161111T074922_N0204_R106_T32DPH_20161111T074922 & Sentinel-2 & $11 / 11 / 2016$ & 2 \\
\hline S2A_MSIL1C_20161114T075922_N0204_R006_T32DNG_20161114T075925 & Sentinel-2 & $14 / 11 / 2016$ & 3 \\
\hline S2A_MSIL1C_20161114T075922_N0204_R006_T32DNH_20161114T075925 & Sentinel-2 & $14 / 11 / 2016$ & 3 \\
\hline S2A_MSIL1C_20161114T075922_N0204_R006_T32DPG_20161114T075925 & Sentinel-2 & $14 / 11 / 2016$ & 3 \\
\hline S2A_MSIL1C_20161114T075922_N0204_R006_T32DPH_20161114T075925 & Sentinel-2 & $14 / 11 / 2016$ & 3 \\
\hline LC08_L1GT_165109_20161204_20170317_01_T2 & Landsat 8 & 04/12/2016 & 4 \\
\hline LC08_L1GT_165110_20161204_20170317_01_T2 & Landsat 8 & 04/12/2016 & 4 \\
\hline LC08_L1GT_166109_20161211_20170316_01_T2 & Landsat 8 & $11 / 12 / 2016$ & 5 \\
\hline LC08_L1GT_166110_20161211_20170316_01_T2 & Landsat 8 & $11 / 12 / 2016$ & 5 \\
\hline S2A_MSIL1C_20161217T080922_N0204_R049_T32DNG_20161217T080925 & Sentinel-2 & $17 / 12 / 2016$ & 6 \\
\hline S2A_MSIL1C_20161217T080922_N0204_R049_T32DNH_20161217T080925 & Sentinel-2 & $17 / 12 / 2016$ & 6 \\
\hline S2A_MSIL1C_20161217T080922_N0204_R049_T32DPG_20161217T080925 & Sentinel-2 & $17 / 12 / 2016$ & 6 \\
\hline S2A_MSIL1C_20161217T080922_N0204_R049_T32DPH_20161217T080925 & Sentinel-2 & $17 / 12 / 2016$ & 6 \\
\hline LC08_L1GT_166109_20161227_20170314_01_T2 & Landsat 8 & $27 / 12 / 2016$ & 7 \\
\hline LC08_L1GT_166110_20161227_20170314_01_T2 & Landsat 8 & $27 / 12 / 2016$ & 7 \\
\hline S2A_MSIL1C_20170126T080921_N0204_R049_T32DNG_20170126T080920 & Sentinel-2 & 26/01/2017 & 8 \\
\hline S2A_MSIL1C_20170126T080921_N0204_R049_T32DNH_20170126T080920 & Sentinel-2 & $26 / 01 / 2017$ & 8 \\
\hline S2A_MSIL1C_20170126T080921_N0204_R049_T32DPG_20170126T080920 & Sentinel-2 & $26 / 01 / 2017$ & 8 \\
\hline S2A_MSIL1C_20170126T080921_N0204_R049_T32DPH_20170126T080920 & Sentinel-2 & $26 / 01 / 2017$ & 8 \\
\hline LC08_L1GT_166109_20170213_20170228_01_T2 & Landsat 8 & 13/02/2017 & 9 \\
\hline
\end{tabular}




\begin{tabular}{|c|c|c|c|c|}
\hline LC08_L1GT_166110_20170213_20170228_01_T2 & Landsat 8 & $13 / 02 / 2017$ & 9 & \\
\hline S2A_MSIL1C_20170225T080921_N0204_R049_T32DNG_20170225T080922 & Sentinel-2 & 25/02/2017 & 10 & \\
\hline S2A_MSIL1C_20170225T080921_N0204_R049_T32DNH_20170225T080922 & Sentinel-2 & 25/02/2017 & 10 & \\
\hline S2A_MSIL1C_20170225T080921_N0204_R049_T32DPG_20170225T080922 & Sentinel-2 & 25/02/2017 & 10 & \\
\hline S2A_MSIL1C_20170225T080921_N0204_R049_T32DPH_20170225T080922 & Sentinel-2 & 25/02/2017 & 10 & \\
\hline LC08_L1GT_167109_20170324_20170329_01_T2 & Landsat 8 & 24/03/2017 & 11 & \\
\hline LC08_L1GT_167110_20170324_20170329_01_T2 & Landsat 8 & 24/03/2017 & 11 & \\
\hline $\begin{array}{l}\text { S1B_EW_GRDM_1SSH_20170126T193341_20170126T193445_004023_006F36_234 } \\
\text { F }\end{array}$ & Sentinel-1 & $26 / 01 / 2017$ & $\mathrm{~N} / \mathrm{A}$ & \\
\hline $\begin{array}{l}\text { SETSM_WV02_20160131_10300100502E9A00_103001005041DF00_seg1_2m_v1.0_ } \\
\text { dem.tif }\end{array}$ & REMA & $31 / 01 / 2016$ & $N / A$ & \\
\hline $\begin{array}{l}\text { 51_34_8m (accessed at } \\
\text { http://data.pgc.umn.edu/elev/dem/setsm/REMA/mosaic/v1.1/8m/) }\end{array}$ & REMA & N/A & $\begin{array}{l}\text { REMA } \\
\text { mosaic }\end{array}$ & $8 m$ \\
\hline $\begin{array}{l}\text { 51_35_8m (accessed at } \\
\text { http://data.pgc.umn.edu/elev/dem/setsm/REMA/mosaic/v1.1/8m/) }\end{array}$ & REMA & N/A & $\begin{array}{l}\text { REMA } \\
\text { mosaic }\end{array}$ & $8 m$ \\
\hline $\begin{array}{l}\text { 52_34_8m (accessed at } \\
\text { http://data.pgc.umn.edu/elev/dem/setsm/REMA/mosaic/v1.1/8m/) }\end{array}$ & REMA & N/A & $\begin{array}{l}\text { REMA } \\
\text { mosaic }\end{array}$ & $8 m$ \\
\hline $\begin{array}{l}\text { 52_35_8m (accessed at } \\
\text { http://data.pgc.umn.edu/elev/dem/setsm/REMA/mosaic/v1.1/8m/) }\end{array}$ & REMA & N/A & $\begin{array}{l}\text { REMA } \\
\text { mosaic }\end{array}$ & $8 m$ \\
\hline
\end{tabular}

S.1: A record of all images used in this study, showing the image ID, associated satellite, acquisition date, and image group number. The image group number indicates which group of images the image was mosaicked with.

S.2: GIF of all 11 RGB base images (https://doi.org/10.5446/47526, (Dell et al., 2020a))

S.3: GIF of all 11 area matrices (https://doi.org/10.5446/47524, (Dell et al., 2020b))

S.4: GIF of all 11 depth matrices (https://doi.org/10.5446/47525, (Dell et al., 2020c)) 



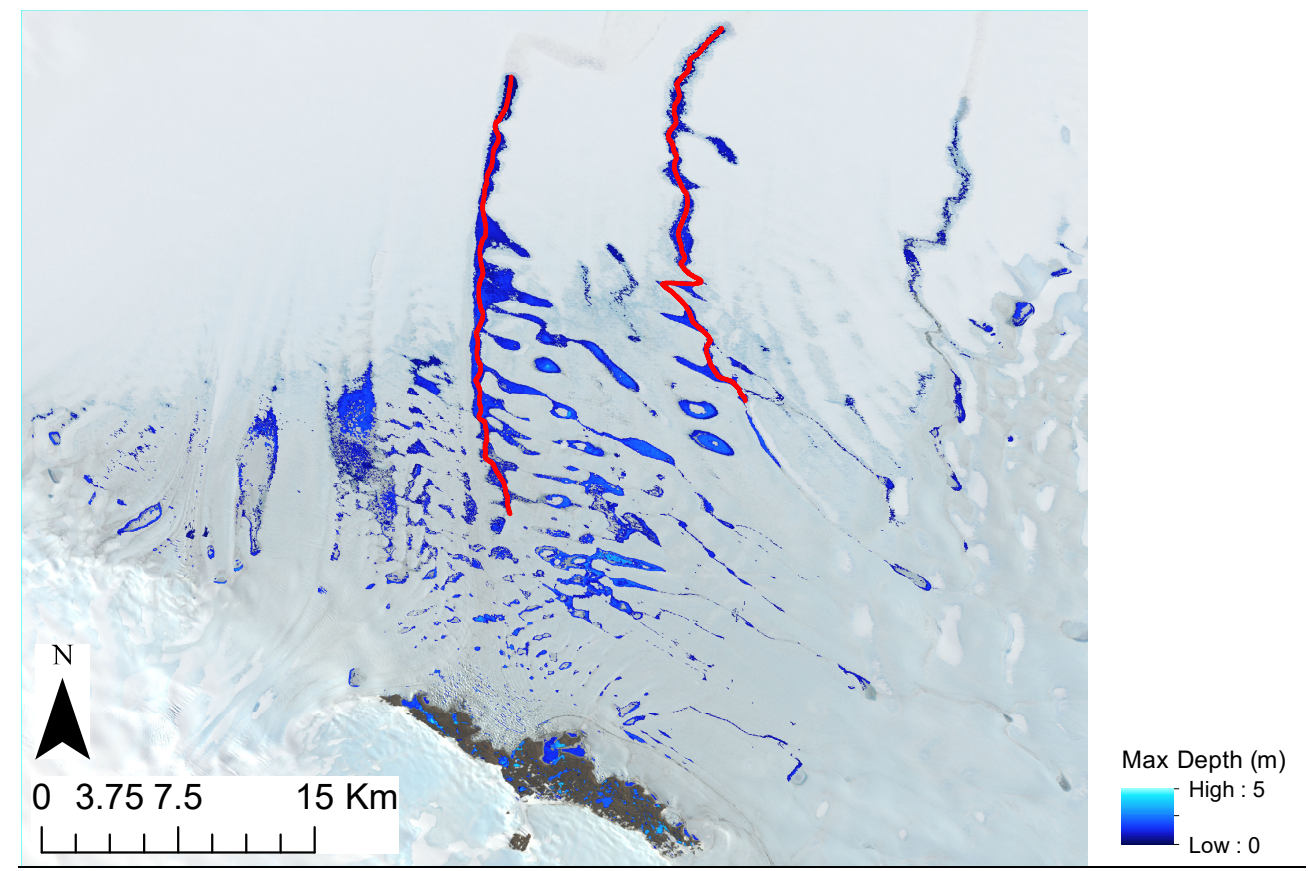

S.5: The paths used for extracting the elevation profiles of the WS and ES (marked by the red lines), overlain on the maximum depth matrix. We used the maximum depth matrix to guide the digitisation of each path, following the deepest water where possible. The base satellite image for the figure was acquired by Landsat 8 on 13 th February 2017. 